\title{
THE EFFECT OF TURBULENCE MODEL VARIATION ON FLAME PROPAGATION OF CNG AS A FUEL IN 4.-VALVE ENGINES
}

\author{
Zoran Jovanovic, Zoran Masonicic
}

\begin{abstract}
In this paper some initial results concerning the evolution of flame propagation of CNG as a fuel in 4-valve engines with tilted valves were presented. Results were obtained by dint of multidimensional modeling of reactive flows in arbitrary geometry with moving boundaries. During induction fluid flow pattern was characterized with organized tumble motion followed by small but clearly legible deterioration in the vicinity of BDC. During compression the fluid flow pattern is entirely three-dimensional and fully controlled by vortex motion located in the central part of the chamber. The effect of turbulence model variation on flame propagation was tackled as well. Namely, some results obtained with eddy-viscosity model i.e. standard $k-\varepsilon$ model were compared with results obtained with $k-\xi-f$ model of turbulence in domain of 4-valve engine in-cylinder flow. Some interesting results emerged rendering impetus for further quest in the near future. In the case of combustion all differences ensuing from turbulence model variation, encountered in the case of non-reactive flow were annihilated entirely. Namely the interplay between fluid flow pattern and flame propagation is invariant as regards both turbulence models applied.
\end{abstract}

Keywords: automotive flows, turbulence modelling

\section{Introduction}

It is known for a long time that various types of organized flows in combustion chamber of IC engines are of predominant importance for combustion particularly with regards to flame front shape and its propagation. Some results related to the isolated or synergic effect of squish and swirl on flame propagation in various combustion chamber layouts are already analyzed and published [1,2] but results concerning the isolated or combined effect of the third type of organized flow i.e. tumble are relatively less presented and sometimes ambiguous [3, 4]. For instance some authors [5] studied the development of swirl and tumble in five different

\section{Zoran Jovanovic}

Institute of Nuclear Science Vinca, University of Belgrade Belgrade, Serbia

Zoran Masonicic

Institute of Nuclear Science Vinca, University of Belgrade Belgrade, Serbia intake valve configurations and found that when both inlet valves are opened no defined tumble flow structure was created rendering quick vortices dissipation before BDC. In spite of the fact that tumble flow is inherent to multivalve engines some authors have demonstrated that some two-valve engines exhibit characteristics similar to tumble flow $[6,7]$. In addition, the fairly similar fluid flow patterns in the vicinity of BDC in various combustion chamber geometries yield entirely different fluid flow patterns, spatial distribution of kinetic energy of turbulence and integral length scales of turbulence in the vicinity of TDC [8]. In such occasions the significance of organized tumble flow is fairly relative. Some theoretical and experimental results show that tumble is of prime importance for specific power and fuel economy increase in modern engines with multi-valve systems. The beneficial effects of tumble on $\mathrm{CO}, \mathrm{CH}$ and $\mathrm{NOx}$ were also demonstrated. From the theory of turbulence is known that vortex filament subjected to compression reduces its length and promotes rotation around its axis yielding the movement on the larger scale ("spin-up" effect). It can be presumed that tumble pursues the same rule i.e. the destruction of formed and expressive tumble during compression stroke generates the higher turbulence intensity and larger integral length scale of turbulence in the vicinity of TDC contributing to the flame kernel formation period reduction and faster flame propagation thereafter. The aforementioned logic imposes the conclusion that the most beneficial fluid flow pattern in the vicinity of BDC is well shaped high intensity tumble. Some additional objectives in this paper were qualitative and quantitative characterization of fluid flow pattern during induction and compression in a particular 4-valve engine, the analysis of the valve/port assembly from the point of compliance with presumed ideal fluid flow pattern and the clout of turbulence model variation on fluid flow and turbulence parameters.

\section{Model and computational method}

The analysis of this type is inherent to multidimensional numerical modeling of non-reactive fluid flow and therefore it is quite logical to apply such a technique particularly due to fact that it is the only technique that encompasses the valve/port geometry layout in an explicit manner. In lieu of the fact that, in its essence, multidimensional models require initial and boundary conditions only their applications is fairly complicated and imply some assumptions and simplifications [9]. The full 
3D conservation integral form of unsteady equations governing turbulent motion of non-reactive mixture of ideal gas is solved on fine computational grid with moving boundaries (piston and valves) in physical domain (500.000-980.000 cells) by dint of AVL FIRE code [10]. In this case the numerical solution method is based on a fully conservative finite volume approach. All dependent variables such as momentum, pressure, density, turbulence kinetic energy, dissipation rate, and passive scalar are evaluated at the cell center. A second-order midpoint rule is used for integral approximation and a second order linear approximation for any value at the cell-face. A diffusion term is incorporated into the surface integral source after employment of the special interpolation practice. The convection is solved by a variety of differencing schemes (upwind or donor cell, interpolated donor cell, quasi second order differencing, central differencing, MINMOD and SMART). The rate of change is differenced by using implicit schemes i.e. Euler implicit scheme and three time level implicit scheme of second order accuracy. The overall solution procedure is iterative and is based on the Semi-Implicit Method for PressureLinked Equations algorithm (SIMPLE). For the solution of a linear system of equations, a conjugate gradient type of solver (CGS) is used. Two different model of turbulence were used. The first one is nearly forty years old $\mathrm{k}-\varepsilon$ model based on Boussinesq's assumption which is certainly the most widely used model for engineering computations. On the contrary to some other models, such as Reynolds-stress closure model [11], its implementation is numerically robust due to simplicity of the model and at the same provides an acceptable level of accuracy for particular applications.

The second one is relatively recent $k-\xi-f$ model of turbulence i.e. eddy-viscosity model based on Durbin's elliptic relaxation concept $[12,13,14]$. This model solves a transport equation for the velocity scale ratio $\xi$ instead of imaginary turbulent normal stress component. In addition, the pertinent hybrid boundary conditions were applied.

\section{Results and discussion}

The analysis of fluid flow pattern during induction and compression was based on a fairly complicated geometry layout presented in figures 1 and 2. Obviously, combustion chamber is constrained with dual intake and exhaust valves. The basic block data sheet consists of bore/stroke ratio $=80 / 81.4 \mathrm{~mm}$, squish gap $=1.19 \mathrm{~mm}$, engine speed $\mathrm{RPM}=5500 \mathrm{~min}^{-1}$ and mixture quality $\lambda=1$. It should be stated that maximum valve lift is $6.95 \mathrm{~mm}$ for intake valves and $6.63 \mathrm{~mm}$ for exhaust valves while the other geometrical data (relative location, valve shape etc.) could be seen in fig. 1 and 2 .

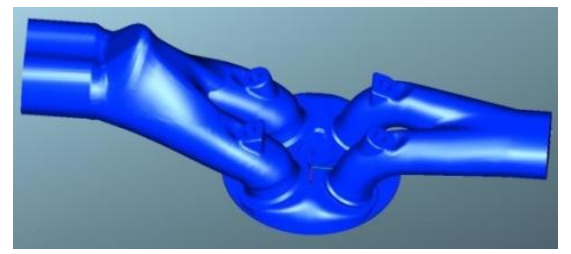

Figure 1. Perspective view of the combustion chamber geometry layout with 4-valves (upper view)

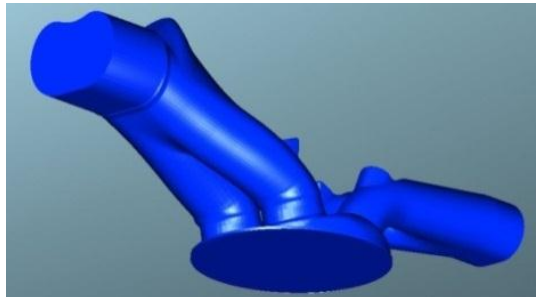

Figure 2. Perspective view of the combustion chamber geometry layout with 4 -valves (bottom view)

The effects of turbulence model variation on the evolution of fluid flow pattern and spatial distribution of kinetic energy of turbulence in 4-valve engine were presented in figs. 3-14 below. Namely, figs. 3, 6, 9 and 12 are related to standard $\mathrm{k}-\varepsilon$ model of turbulence while figs. $4,7,10$ and 13 are related to $\mathrm{k}-\xi$-f model of turbulence. Due to symmetry in $\mathrm{x}-\mathrm{y}$ plane results for both model of turbulence were presented in the same figure (figs. 5, 8, 11 and 14). In order to alleviate comparisons of fluid flow patterns particularly in the case of subtle differences colors were employed as well.

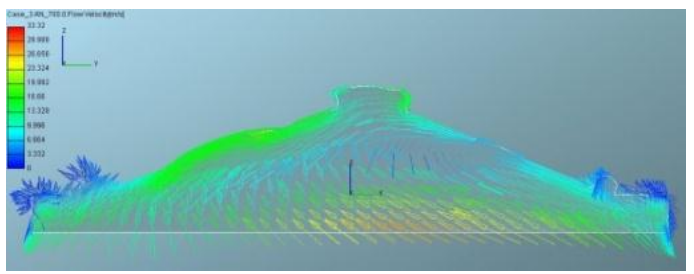

Figure 3. Fluid flow pattern in $\mathrm{x}-\mathrm{z}$ plane, $\mathrm{y}=$ const.at $340 \mathrm{deg}$. ATDC, $\mathrm{k}-\varepsilon$

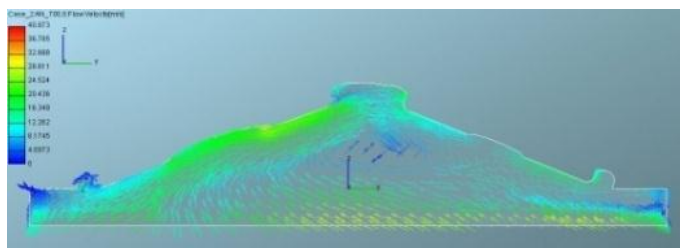

Figure 4. Fluid flow pattern in $\mathrm{x}-\mathrm{z}$ plane, $\mathrm{y}=$ const.at $340 \mathrm{deg}$. ATDC, k$\xi-\mathrm{f}$

During induction and large portion of compression stroke (up to $270 \mathrm{deg}$. ATDC) no legible differences as regards the evolution of fluid flow pattern and spatial distribution of kinetic energy of turbulence were observed and therefore not presented due to economy of the paper. The significant differences are commencing in the vicinity of TDC.

Namely, the fluid flow pattern and less intensive colors in figs. 4, 7, 5 (right) and 8(right) than in figs. 3, 6, 5 (left) and 8 (left) indicate less expressive vortex flow and generally smaller velocities in the case of $\mathrm{k}-\xi$-f model of turbulence yielding somehow the detention of vortex displacement to the exhaust valve zone thereafter. Larger velocities in the case of $\mathrm{k}-\varepsilon$ model of turbulence are encountered in x-y plane as well (figs. 5 and 8, left).

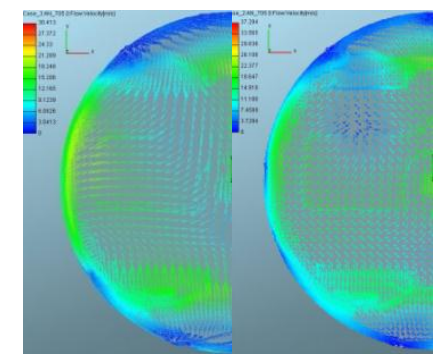


Proc. of the Sixth International Conference on Advances in Mechanical and Robotics Engineering - AMRE 2017.

Copyright (C) Institute of Research Engineers and Doctors. All rights reserved.

ISBN: 978-1-63248-140-5 doi: 10.15224/ 978-1-63248-140-5-42

Figure 5. Fluid flow pattern in $x-y$ plane, $z=$ const.at 345 deg. ATDC, $k$ $\varepsilon$ (left) and $\mathrm{k}-\xi$-f(right)

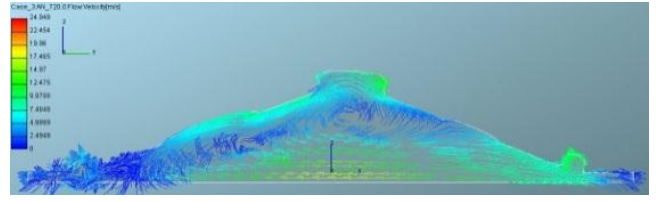

Figure 6. Fluid flow pattern in $\mathrm{x}-\mathrm{z}$ plane, $\mathrm{y}=$ const.at $360 \mathrm{deg}$. ATDC, k-

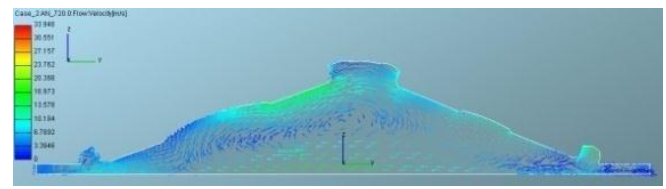

Figure 7. Fluid flow pattern in $\mathrm{x}-\mathrm{z}$ plane, $\mathrm{y}=$ const.at $360 \mathrm{deg}$. ATDC, $\mathrm{k}$ $\xi-\mathrm{f}$

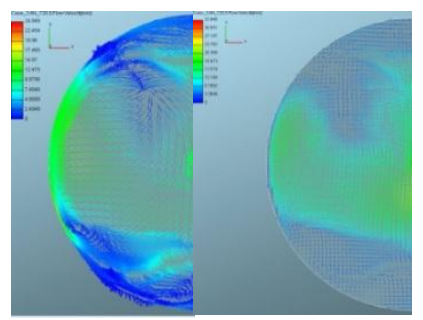

Figure 8. Fluid flow pattern in $x-y$ plane, $z=$ const.at 360 deg. ATDC, $k-$ $\varepsilon$ (left) and $\mathrm{k}-\xi$-f(right)

Differences in fluid flow patterns are pursued in a straightforward fashion by certain differences in turbulence intensity and spatial distribution of kinetic energy of turbulence in all planes. It can be seen that in the case of $\mathrm{k}-\varepsilon$ model of turbulence the maximum kinetic energy of turbulence is located in the central part of the chamber (figs. 9 and 12) while in the case of $\mathrm{k}-\xi$-f model of turbulence the maximum kinetic energy of turbulence is shifted to the intake valve zone (figs. 10 and 13). In addition, in the case of $\mathrm{k}-\varepsilon$, high values of kinetic energy of turbulence prevail and engulf nearly the entire chamber (figs. 11 and 14, left) while in the case of $\mathrm{k}-\xi$-f these zones are obviously smaller and akin to characteristic bean-like form (figs. 11 and particularly 14, right). Such behavior could largely affect all other in-cylinder processes that incur such as mixing, combustion and etc. In general $\mathrm{k}-\varepsilon$ model of turbulence generates higher values of kinetic energy of turbulence over the broader part of the chamber. Namely k- $\varepsilon$ over predicts its value.

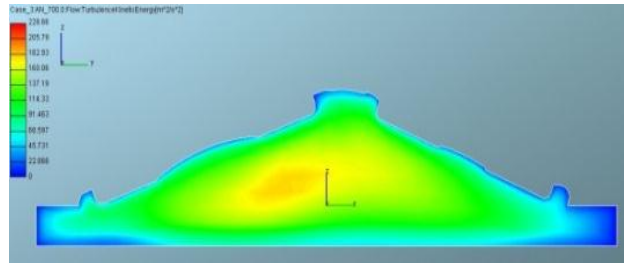

Figure 9. Spatial distribution of kinetic energy of turbulence in $x-z$ plane, $\mathrm{y}=$ const. at $340 \mathrm{deg}$. ATDC, $\mathrm{k}-\varepsilon$

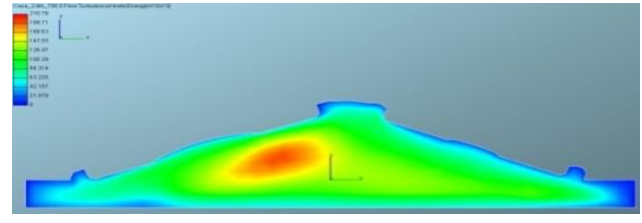

Figure 10. Spatial distribution of kinetic energy of turbulence in $\mathrm{x}-\mathrm{z}$ plane, $\mathrm{y}=$ const. at $340 \mathrm{deg}$. ATDC, $\mathrm{k}-\xi-\mathrm{f}$

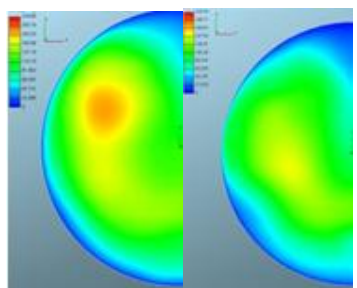

Figure 11. Spatial distribution of kinetic energy of turbulence in $x-y$ plane, $\mathrm{z}=$ const. at $340 \mathrm{deg}$. ATDC, $\mathrm{k}-\varepsilon$ (left) and $\mathrm{k}-\xi$-f(right)

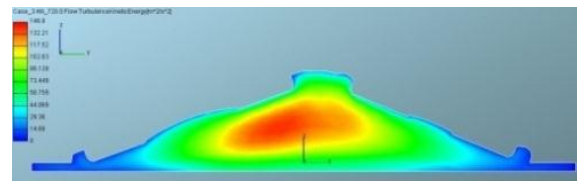

Figure 12. Spatial distribution of kinetic energy of turbulence in $\mathrm{X}-\mathrm{Z}$ plane, $\mathrm{y}=$ const. at $360 \mathrm{deg}$. ATDC, $\mathrm{k}-\varepsilon$

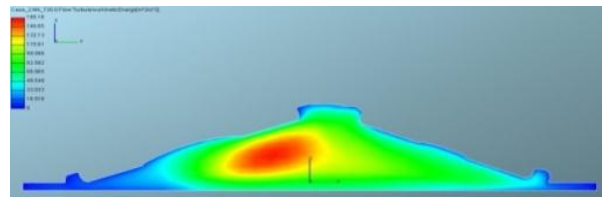

Figure 13. Spatial distribution of kinetic energy of turbulence in $\mathrm{x}-\mathrm{z}$ plane, $y=$ const. at $360 \mathrm{deg}$. ATDC, $\mathrm{k}-\xi-\mathrm{f}$

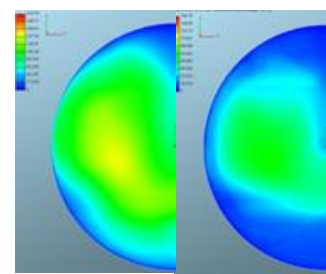

Figure 14. Spatial distribution of kinetic energy of turbulence in $x-y$ plane, $\mathrm{z}=$ const. at $360 \mathrm{deg}$. ATDC, $\mathrm{k}-\varepsilon$ (left) and $\mathrm{k}-\xi$-f(right)

The fairly interesting results were obtained in the case of combustion that was tackled as well. Namely, combustion was modeled in an eclectic, theoretically and experimentally validated eddy-breakup model $[9,10]$. In the case of combustion all the subtleties as regards fluid flow pattern and spatial distribution of kinetic energy of turbulence due to turbulence model alteration, observed in figs. 3-14, were annihilated entirely.

The fluid flow pattern and flame propagation (represented as iso-contours of temperatures) in various cut planes, for two different models of turbulence (k- $\varepsilon$ and $\mathrm{k}-\xi-\mathrm{f}$ ) were presented in figs. $15-22$, below.

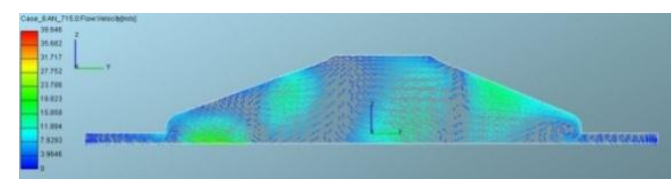

Figure 15. Fluid flow pattern in $\mathrm{x}-\mathrm{z}$ plane, $\mathrm{y}=$ const. (0.0) at $355 \mathrm{deg}$. ATDC, $\mathrm{k}-\varepsilon$ 
Proc. of the Sixth International Conference on Advances in Mechanical and Robotics Engineering - AMRE 2017.

Copyright (C) Institute of Research Engineers and Doctors. All rights reserved.

ISBN: 978-1-63248-140-5 doi: 10.15224/ 978-1-63248-140-5-42

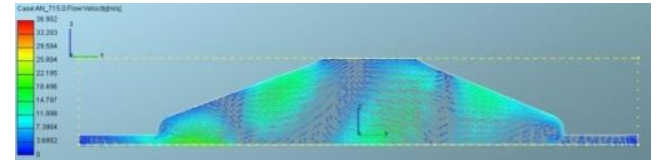

Figure 16. Fluid flow pattern in $\mathrm{x}-\mathrm{z}$ plane, $\mathrm{y}=$ const. (0.0) at $355 \mathrm{deg}$. ATDC, $\mathrm{k}-\xi-\mathrm{f}$

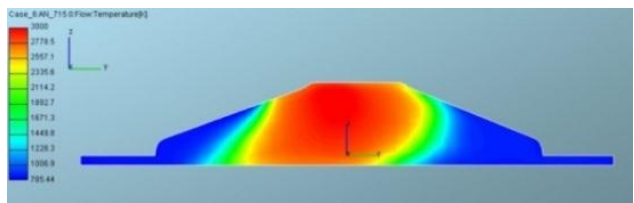

Figure 17. Spatial distribution of temperature in $\mathrm{x}-\mathrm{z}$ plane, $\mathrm{y}=$ const. $(0.0)$ at 355 deg. ATDC, $\mathrm{k}-\varepsilon$

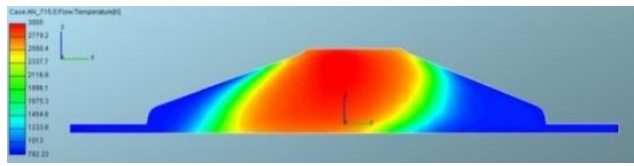

Figure 18. Spatial distribution of temperature kinetic in $\mathrm{x}-\mathrm{z}$ plane, $\mathrm{y}=$ const. (0.0) at $355 \mathrm{deg}$. ATDC, $\mathrm{k}-\xi-\mathrm{f}$

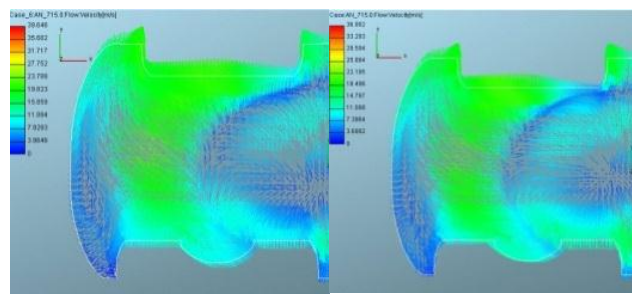

Figure 19. Fluid flow pattern in $x-y$ plane, $z=$ const. (mid-height of the wedge chamber) at $355 \mathrm{deg}$. ATDC, k- $\varepsilon$ (left) and k- $\xi$-f (right)

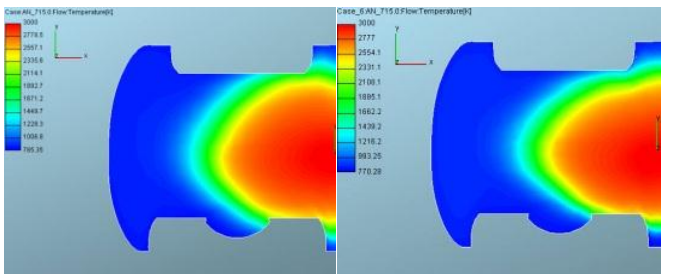

Figure 20. Spatial distribution of temperature in $x-y$ plane, $z=$ const. (midheight of the wedge chamber) at $355 \mathrm{deg}$. ATDC, $\mathrm{k}-\varepsilon$ (left) and k- $\xi-\mathrm{f}$ (right)

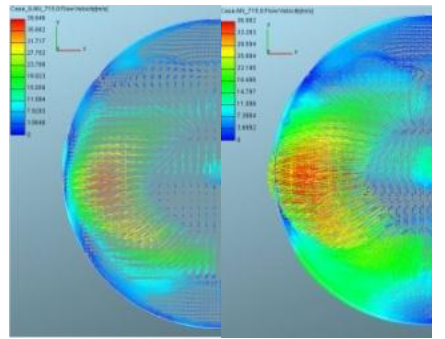

Figure 21. Fluid flow pattern in $x-y$ plane, $z=$ const. (squish zone) at 355 deg. ATDC, k- $\varepsilon$ (left) and k- $\xi-f$ (right)

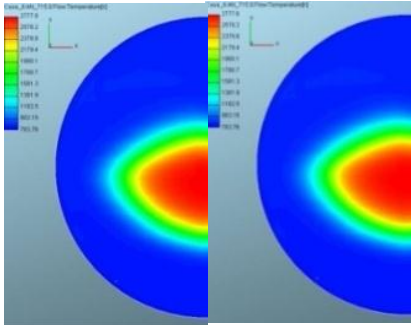

Figure 22. Spatial distribution of temperature in $x-y$ plane, $z=$ const. (squish zone) at $355 \mathrm{deg}$. ATDC, $\mathrm{k}-\varepsilon$ (left) and $\mathrm{k}-\xi$-f (right)

It can be seen, in figs. $15,16,19$ and 21 that, in the case of combustion, no clear difference in fluid flow pattern is observed. Namely, the well-known "flame dominated fluid flow pattern" is encountered characterized with higher velocities in front of the flame front and relaminarisation behind the flame front (figs. 15, 16, 19 and 21). This is legible in all cut planes considered. In comparison with corresponding fluid flow pattern in no combustion case (figs. 6, 7 and 8) clear differences are indicated.

The main reason for the relaminarization of the fluid flow behind the flame front lies in the fact that flame propagation through un-burnt mixture, entire of itself, accelerates the hot gas in front of the flame front. In the case of shear the production of turbulence increases with the effect of flame acceleration thereafter. In the compressed zone in front of the flame front the divergence of the mean velocity is negative yielding the generation of turbulence as well. In addition the sign and the magnitude of the density gradient within the flame affect the diffusion of turbulence. Referring to the energy conservation equation one can find the maximum enthalpy in the zone of minimal density, i.e. behind the flame front so these higher temperatures cause the intensive increase of viscosity with the consequential increase of $\mathrm{Re}_{\mathrm{t}}$-number, the increase of viscous dissipation of turbulence and shifting of the velocity fluctuations to the low frequency part of spectrum.

In the heat release zone the dilatation of turbulence reduces the turbulent kinetic energy yielding fairly legible soothing or attenuation (relaminarization) of the fluid flow. It can be seen, in figs. $17,18,20$ and 22 , that in a particular combustion chamber geometry layout, flame propagation as regards its velocity and its flame front shape is entirely invariant vis-à-vis alteration of $\mathrm{k}-\varepsilon$ model of turbulence to $\mathrm{k}-\xi$-f model of turbulence.

\section{Conclusions}

The fluid flow pattern during induction and compression in the particular combustion chamber geometry of 4-valve engine is extremely complex and entirely three-dimensional. In the case with two valves opened tumble motion during induction is clearly legible and followed by gradual deterioration in the vicinity of BDC due to non-uniform distribution along y-axis. During compression strong vortex flow around y-axis and fairly expressive coinciding flow along $\mathrm{x}$-axis in reverse direction is encountered contributing to the inconvenient spatial distribution of kinetic energy of turbulence in the vicinity of TDC. The modeling of turbulence strongly affects the evolution of fluid flow pattern and spatial distribution of kinetic energy of turbulence in 4-valve engines. In general $\mathrm{k}-\varepsilon$ model of turbulence generates higher values of kinetic energy of turbulence over the broader part of the chamber than corresponding $\mathrm{k}-\xi-\mathrm{f}$ model of turbulence. In the case of combustion all differences ensuing from turbulence model variation, encountered in the case of non-reactive fluid flow were annihilated entirely. Namely the interplay between fluid 
flow pattern encountered ("flame dominated fluid flow") and flame propagation is invariant as regards both turbulence models applied. On the contrary, such a conclusion is not valid either in the case of "squish dominated flows" or in the case of "coincident flow".

\section{References}

[1] [1] J.Danneman, K.Pielhop, M. Klaas, W. Schroeder(2010) „Cycle resolved multi planar flow measurements in a four valve combustion engine", Exp.Fluids, Research article, DOI 10.1007/s00348-010-0963-4

[2] [2] Z. Jovanovic, S. Petrovic "The mutual interaction between squish and swirl in IC Engines", (1997) Mobility and Vehicle Mechanics 23, 3, 72-86

[3] [3] K. Lee, C. Bae, K. Kang "The effects of tumble and swirl flows on flame propagation in a four-valve S.I.engine", Applied Thermal Engineering 27 (2007) 2122-2130

[4] [4] G.J.Micklow, W. D. Gong "Intake and in cylinder flow field modeling of a four valve diesel engine" Proc.IMechE(2007) vol. 221, Journal of Automobile Engineering, 1425-1440

[5] [5] B. Khaligi "Intake generated swirl and tumble motion in a 4.valve engine with various intake configurations" SAE Paper 900059

[6] [6] Z.Jovanovic, S. Petrovic, M. Tomic "The effect of combustion chamber geometry layout on combustion and emission" (2008) Thermal Science vol.12, No.1, pp. 7-24

[7] [7] Z. Jovanovic, Z.Masonicic, M. Tomic „The vice-verse movement of the reverse tumble centre of rotation in a particular combustion chamber", MTM Machines Technologies Materials, Year II, issue 6-7, (2008) ISSN 1313-02261, pp. 17-20

[8] [8] Z. Masonicic, Z. Jovanovic "The effect of combustion chamber geometry layout variations onto fluid flow pattern", International Automotive Conference with Exhibition, SCIENCE AND MOTOR VEHICLES, NMV0774, Belgrade, 2007, ISBN 978-86-80941-31-8

[9] [9] Z.Jovanovic "The role of tensor calculus in numerical modeling of combustion in IC engines" Computer Simulation in Fluid Flow, Heat and Mass Transfer and Combustion in Reciprocating Engines, Hemisphere Publishers (1989) 457-542, ISBN 0-89116-392-1

[10] [10] CFD Solver, AVL FIRE 2009.1

[11] [11] C.G. Speciale, S. Sarkar, T. B. Gatski „Modelling the pressure strain correlation of turbulence - an invariant dynamical system approach“, pp.1-51, ICASE Report No. 90-5, 1990

[12] [12] K.Hanjalic, M.Popovac, M.Hadjiabdic „A robust near-wall elliptic relaxation eddy viscosity turbulence model for CFD", International Journal of Heat and Fluid Flow, 25(2004) 1047-1051

[13] [13] P.A.Durbin „Near wall turbulence closure modeling without damping functions“, Theor.Comput. Fluid Dynamics (1991) 3 1-13

[14] [14] B.Hanjalic "Blending the RANS and LES concepts for computing industrial and environmental flows: a perspective", SCIENCE AND MOTOR VEHICLES, NMV11AutoSim01, Belgrade, 2011, ISBN 978-86-80941-36-3

[15] [15] B.Basara, Z. Pavlovic "PANS vs. RANS for computation of the flow through intake engine ports", International Automotive Conference with Exhibition, SCIENCE AND MOTOR VEHICLES, NMV11 AutoSim09, Belgrade, 2011, ISBN 978-86-80941-36-3

[16] [16] M.Popovac, K. Hanjalic „Compound wall treatment for RANS computation of complex turbulent flows and heat transfer", Flow, Turbulence, Combustion (2007) 78:177-202 